\title{
Item distribution, internal consistency, and structural validity of the German version of the DEMQOL and DEMQOL-proxy
}

\author{
Christian G. G. Schwab ${ }^{1,2}$, Martin Nikolaus Dichter ${ }^{1,2^{*}}$ and Martin Berwig ${ }^{3,1}$
}

\begin{abstract}
Background: Accurate assessment of health-related quality of life as an endpoint in intervention studies is a major challenge in dementia research. The DEMQOL (29 items) and the proxy version (32 items), which is partly based on the $D E M Q O L$, are internationally used instruments. To date, there is no information on the structural validity, item distribution, or internal consistency for the German language version of these questionnaires.

Methods: This psychometric study is based on a secondary data analysis of a sample of 201 outpatients with a mild form of Alzheimer's disease (AD) and their informal caregivers. The informal caregivers who were interviewed were involved in the care of the person with AD several times per week. The analysis for the evaluation of the structural validity was performed using Mokken scale analysis. The internal consistency was calculated using the $\rho$ of the Molenaar Sijtsma statistic and Cronbach's a.

Results: For both versions, four subscales were identified: [A] "positive emotions", [B] "negative emotions", [C] "physical and cognitive functioning", and [D] "daily activities and social relationships". For both instruments, the internal consistency of all subscales was considered "good" ( $\rho=0.71-0.88, a=0.72-0.87$ ).

Conclusions: The results are a first indication of good construct validity of the instruments used for the German setting. We recommend further investigations of the test-retest reliability and the inter-rater reliability of the proxy instrument.
\end{abstract}

Keywords: Dementia, Quality of life, Structural validity, Internal consistency

\section{Background}

According to the World Alzheimer Report, a person was diagnosed with dementia every $3.2 \mathrm{~s}$ in 2015 . Currently, approximately 46.8 million people worldwide are living with dementia [1]. Dementia is a neurocognitive disorder associated with a significant cognitive decline from a previous level of performance, resulting in a dependency on others to perform activities of daily living [2].

Health-related quality of life (HRQoL) reflects an important desire for persons living with dementia and is therefore used as a general endpoint in many interventional studies. Additionally, HRQoL is increasingly used for assessments of anti-dementia drugs and by the European Medicines

\footnotetext{
* Correspondence: Martin.Dichter@dzne.de

${ }^{1}$ German Center for Neurodegenerative Diseases (DZNE), Stockumer Straße 12, 58453 Witten, Germany

${ }^{2}$ School of Nursing Science, Witten/Herdecke University, Stockumer Straße

12, 58453 Witten, Germany

Full list of author information is available at the end of the article
}

Agency to determine the benefits of such drugs [3]. HRQoL in persons living with dementia also is also considered by regulatory authorities and administrative agencies who must judge this parameter based on a resident's degree of self-sufficiency. Furthermore, HRQoL is used in economic evaluations of persons in all stages of dementia [4-6].

HRQoL is defined by Hays and Reeve as how well a person functions in his/her life and perceives his/her well-being in the physical, mental, and social domains of health [7]. In this definition, functioning refers to the individual's ability to achieve predefined activities, and well-being refers to individual's subjective feelings $[8,9]$. Based on this and similar definitions of HRQoL, Karimi and Brazier [8] conclude that HRQoL is a particular type of health description; therefore, the World Health Organization (WHO) defines health as "a state of complete physical, mental and social well-being, and not merely the absence of disease and infirmity" [10]. This definition indicates that $\mathrm{HRQOL}$ measurements reflect 
health in a wider sense (i.e., well-being and functioning) than solely other clinical outcomes (e.g., 5-year survival rate, rate of restenosis, death, and tumor recurrence).

According to Bakas et al. [11], three models of HRQoL are frequently used: the WHO [12] International Classification of Functioning, Disability and Health (ICF) Model of Functioning and Disability, the HRQoL Model from Wilson and Cleary [9], and, based on this model, the quality of life (QoL) measurement by Ferrans et al. [13].

Smith et al. observed the need to develop a conceptual framework that addressed the differences between the views expressed about HRQoL by people with dementia and their caregivers [14]. The results of the literature analysis and the findings from expert opinions could also be verified by data from interviews of individuals with dementia and their family caregivers [14]. Thus, an empirical justification of the conceptual framework can be assumed. Based on the five domains of the conceptual framework ("health and well-being", "cognitive functioning", "daily activities", "social relationships", and "selfconcept"), they developed two interviewer-administered instruments called Dementia Quality of Life (DEMQOL) and its proxy version (DEMQOL-Proxy). The authors conducted a pretest factor analysis during the development of the instrument (DEMQOL $n=130, D E M Q O L$ Proxy $n=126)$ that covered the same four dimensions: "positive emotions", "negative emotions", "memory", and "daily activities". For the DEMQOL-Proxy, a two-factor solution, as given by "emotion" and "functioning", has been suggested by the results of the pretest. The factors of both the self-report and proxy version, however, did not fully support the original conceptual framework [15]. Mulhern and colleagues [4] published a factor analysis with a sample of 644 persons with mild to moderate dementia and 682 proxies. In their study, the subscales "cognition", "positive emotion" and "negative emotion" could be used on both instruments [4]. However, "social relationships" and "loneliness" were observed only in the $D E M Q O L$, while the subscales "daily activities" and "appearance" only occurred in the DEMQOL-Proxy [4].

The DEMQOL consists of 28 items, while the DEMQOL-Proxy includes 31 items on a four-point Likert-type scale with the following responses: a lot, quite slightly, a little, and not at all. The scale includes an additional global QoL item (item 29 resp. 32). Items were scored from 1 to 4 , with higher scores indicating better HRQoL. It must be noted that there are five contraindicative items in the DEMQOL and the DEMQOL-Proxy ( $4=$ a lot, ..., $1=$ not at all). The global QoL item also contraindicates the answer options of "very good", "good", "fair", and "poor". Fifteen items, in addition to the global QoL item, are similar in both versions of the DEMQOL; however, there are also items that are not part of the other instrument [15].
The $D E M Q O L$ can be used in mild to moderate dementia as a self-report form and also for severe dementia in a proxy version (DEMQOL-Proxy) across different types of dementia and care arrangements [15]. The utility score $(D E M Q O L-U)$, which is created from a subset of items from the $D E M Q O L$, can also be used for economic assessments [4]. The DEMQOL instrument was developed and tested in the UK, which was reported in a Health Technology Assessment (HTA) report [15]. Consequently, it is used more frequently in the UK. While there is a German translation, the results of a linguistic validation have not been reported [16]. No adequate results are available for psychometric testing of the German versions, which are required for both research and applied purposes [17]. To date, the DEMQOL has been subjected to at least four more latent variable modeling investigations in two countries since its foundation work [15], two factor analyses [4, 18], bifactor modeling [19], and Rasch modeling [20].

This paper consequently targets the first evaluation of the item distribution, structural validity as a part of the construct validity, and internal consistency of the German version of both the DEMQOL and DEMQOL-Proxy. For this purpose, a parallel iterative Mokken scale analysis (MSA) is used as a further procedure in addition to the aforementioned methods.

\section{Methods}

The analysis for the present study was performed on a secondary data analysis using anonymous baseline data of a randomized controlled trial called the Cognitive Rehabilitation and Cognitive Behavioral Treatment for Early Dementia in Alzheimer's Disease (CORDIAL) study [21]. To test the structure found in terms of a sensitivity analysis, we used the data of the follow-up surveys after three $\left(T_{1}\right)$ and after nine months $\left(T_{2}\right)$. The CORDIAL study was performed to provide clinically meaningful benefits and to evaluate the feasibility, acceptance, efficacy, and usefulness of interventions in cognitive rehabilitation. The study was accomplished as a multicenter randomized controlled trial on persons living with Alzheimer's disease (AD) and their informal caregivers (as proxy raters). Ethical clearance was granted for the CORDIAL study by the Ethics Commission of the Faculty of Medicine of the Technical University of Munich on 12/10/2009 under the number 2113/ $08 \mathrm{~S}$. We have refrained from re-auditing as ethical clearance is not required for analyses based on secondary data [22] or for studies using anonymous data.

\section{Setting and participants}

The baseline data of the CORDIAL study were collected from July 2008 to September 2009. The first inclusion criterion required participants to be elderly outpatients 
with an established ICD-10 diagnosis of AD with mild severity, as defined by a Mini-Mental State Examination (MMSE) score of 21 or above. A differential diagnosis to other forms of dementia was conducted by the recruitment centers. This process was completed to obtain a similar picture of symptoms of the participants, in which memory problems in the early stage are the focus of their everyday problems. Patients were recruited from the ten recruitment centers of the study, including memory clinics and neurological and psychotherapeutic practices throughout Germany.

The need for a designated informal caregiver who was involved in the care of the person living with AD several times a week was the second inclusion criterion for the study. Exclusion criteria were comprised of acute psychiatric or physical disorders, ongoing formal psychotherapy or cognitive training, regular visits to day care facilities, an impending hospital or nursing home admission, a poor command of the German language, alcohol or substance dependence, and participation in another interventional trial. Stable doses of cholinesterase inhibitors, memantine, nootropics, antidepressants, and antipsychotics were permissible as concomitant medications of the person living with $\mathrm{AD}$ [21].

\section{Procedures}

The task of the recruitment centers was to inform possible study participants in advance of the study. This process was completed through personal conversations and informational materials. Written informed consent was obtained from both, persons living with $\mathrm{AD}$ and their informal caregivers. Independent psychologists, serving as raters, conducted the assessments of the CORDIAL study. The raters received a one-day seminar with case studies for use during the assessments, including the DEMQOLquestionnaires. The two interviews with the persons living with $\mathrm{AD}$ and the informal caregivers were completed separately. Thus, the informal caregivers were blinded to the answers of the persons living with AD. The monitoring of the study was conducted by an interdisciplinary data monitoring and safety board.

\section{Measurements}

In addition to the previously described $D E M Q O L$ and DEMQOL-Proxy, which are the main topics of interest for our study, further instruments were used. To determine the cognitive ability of the persons living with $\mathrm{AD}$, the German version of the MMSE was used [23]. It is an eleven-question assessment covering five areas of cognitive functioning: orientation, registration, attention and calculation, recall, and language. Each of the 30 tasks is evaluated using a point (range of total scores: 0 to 30). A lower MMSE score indicates a more severe cognitive impairment. To assess impairment of activities of daily living among persons living with $\mathrm{AD}$, the Bayer Activities of Daily Living Scale (B-ADL) by Hindmarch et al. [24] was used. The B-ADL displays a proxy rating assessment for elderly persons with loss of cognitive performance. It is comprised of 25 items rated on a 10 -point scale $(1=$ never, $\ldots, 10=$ always $)$. The total scores range from 1 to 10 , and higher scores indicate higher impairment. To evaluate depressive symptoms, the long form of the Geriatric Depression Scale (GDS) from Yesavage [25] was used. This assessment has 30 dichotomous items (yes or no; directed differently); thus, the total score ranges from 0 to 30 points (higher scores reflect more severe depression). Finally, the Neuropsychiatric Inventory (NPI) by Cummings [26] was used to characterize the neuropsychiatric symptoms and psychopathology of persons living with AD. The NPI covers twelve types of neuropsychiatric disturbances. The frequencies of the symptoms are rated on a 4-point scale multiplied by their severities on a 3-point scale. A higher score (0 to 144) indicates more challenging behavior.

For a description of the informal caregiver, two assessments were used. First, the Beck Depression Inventory (BDI) from Hautzinger et al. [27] is an instrument that assesses the severity of depression. For each of the 21 questions, there are four different answers that are arranged according to their intensity (e.g., item 1: $0=\mathrm{I}$ do not feel sad; ..., $3=\mathrm{I}$ am so sad or unhappy that I cannot stand it). A higher BDI score (range for total scores: 0 to 63) indicates greater severity of depressive symptoms. Second, the full Zarit Burden Interview (ZBI-22; 22 items) by Zarit et al. [28] measures the subjective burden of informal caregivers, associated with functional/behavioral impairments and the home care situation. The ZBI-22 is rated on a 5 -point scale $(0=$ never, ..., $4=$ nearly always), and higher scores (range for total scores: 0 to 88 ) indicates a higher burden.

The socio-demographic data collected for the informal caregiver included age in years and sex, and for the person living with $\mathrm{AD}$, education in years was recorded. In addition, the informal caregiver was asked to explain his/her relationship to the person living with $\mathrm{AD}$.

\section{Statistical analysis}

The descriptions of the participants, missing data, and item distribution were conducted using descriptive statistics. The analysis of the item difficulty was based on the proportion of responses endorsing the best and worst ratings (i.e., ceiling/floor effects). A corresponding effect was assumed conservatively, as long as the mean value of the item was in the lower end (upper 20\%) of the respective item range.

For the analysis of the structural validity of the $D E M$ $Q O L$ and DEMQOL-Proxy, as part of the construct validity, we used the MSA. The MSA is a useful tool for 
researchers who wish to construct unidimensional tests or use questionnaires that comprise multiple binary or polytomous items and enable the examination of reliability without the use of Cronbach's alpha [29]. The MSA is a method of the non-parametric item response theory originating from assumptions of the unidimensionality of tests or scales, local independency and monotonicity [30]. The method is established in the context of scale development and has been widely used in QoL research [31, 32].

The MSA provides additional information about the relationship between items. As an indicator of the internal correlation of each subscale, the MSA uses Loevinger's $\mathrm{H}$ coefficient $\left(\mathrm{H}^{\mathrm{S}}\right)$. According to Sijtsma and Molenaar [33], the following interpretation of $\mathrm{H}^{\mathrm{S}}$ scores was applied to describe the scale: $>0.5=$ "strong", $>0.4$ = "medium", and > $0.3=$ "weak". The correlation of a single item to the other items of the scale is expressed by the value $\mathrm{H}_{\mathrm{i}}$. The $\mathrm{H}_{\mathrm{i}}$ should be non-negative for the Mokken model to hold. Depending on the source, an $\mathrm{H}_{\mathrm{i}}$-value from 0 to 0.55 is recommended. We have fixed the $\mathrm{H}_{\mathrm{i}}$-value to the typically used minimum of $>0.3$. Items that fall below this level have weak discrimination power and are not useful for this scale. The $\mathrm{H}_{\mathrm{ij}}$ designated the coefficient of two items.

The criterion (Crit) of the MSA by Molenaar and Sijtsma [34] was used to identify items that partially satisfy the assumptions for monotonous homogeneity or double monotonicity. For each item, this diagnostic value combines the $\mathrm{H}$ coefficient, the frequency and size of the violations, and their significance. Every item should have a Crit value of less than 40 , and optimally a Crit value of 0 . A Crit value of greater than 80 displays a strong indication that an item has violated the assumption for the MSA in this subscale. The critical values were calculated separately for each of the ten imputed records (see below). As a result, individual injuries of double monotonicity should not be systematically increased by a factor of ten.

For the exploratory investigation of the instruments, a method of parallel iteration was used, which consists of two steps. In the first step, cores were determined. The cores are items in an item pool that are strongly correlated with each other $\left(\mathrm{H}_{\mathrm{ij}}\right)$ and are less correlated with the other cores when examined as a dyad. This finding means that other items from the pool could improve the $\mathrm{H}^{\mathrm{S}}$ value in a similar way that a second core could. Otherwise, the weaker second core was returned into the item pool as single items. As a strong correlation for a core, we defined a minimum $\mathrm{H}_{\mathrm{ij}}$-value of 0.45 as a reference according to Müller-Schneider [35]. Analogous to the procedure in a factor analysis, the number of subscales is thereby predefined. In the second step, an iterative MSA was performed in parallel for each core determined. All items were tested in parallel. Accordingly, all remaining items would be tested against any core, and the item with the highest $\mathrm{H}_{\mathrm{i}}$-value to a specific core would be chosen. In doing so, the assignment of an item should not lead to a violation of monotonicity (Crit > 40). This procedure was used with regard to content in the case of a possible allocation to two different cores (cross loader). The search procedure stopped when there were no further items that fulfilled the requirements $\left(\mathrm{H}_{\mathrm{i}} \geq 0.3\right.$ or Crit $>40$ ) or when all the items had been incorporated into a scale. This method of parallel iterative analysis allowed for the identification of smaller subscales with higher $\mathrm{H}^{\mathrm{S}}$ values, as opposed to the Automated Item Selection Procedure (AISP) for MSA [36].

As a precondition, the MSA assumes only complete cases and integers as values. Therefore, missing values may have to be imputed. In the case of instrument testing, however, the imputation of missing data should be performed with caution. We used a two-way imputation, which is a Bayesian method for estimating missing data in tests and questionnaires [37].

The internal consistency was assessed with the coefficient rho $(\rho)$ of the Molenaar Sijtsma statistic. The $\rho$ coefficient is not as prone to bias as Cronbach's $\alpha$ and should therefore be preferentially used [38, 39]. For comparison purposes, we also calculated Cronbach's alpha $(\alpha)$. Values for a $\rho$ or $\alpha$ between 0.70 and 0.95 indicated "good" internal consistency [40]. Finally, we conducted a part-whole-corrected item-total correlation $\left(r_{i t}\right)$ calculation. For this purpose, the coefficient for the item to be examined against the scale without this item was computed. Items with $r_{i t}$ coefficients $>0.5$ reflected a "high" correlation, and those with $r_{i t}>0.3$ reflected a "moderate" correlation [41]. An $\mathrm{r}_{\mathrm{it}}$ correlation of 0.3 and below indicated that the item did not correlate well with the scale as the item may not measuring the same construct as the other variables.

All analysis were performed using $\mathrm{R}$ environment for statistical computing version 3.4.1 (30.06.2017) [42]. The following packages were used for the MSA and the imputation, respectively: "mokken" version 2.8.6 [43, 44] and "miceadds" version 2.5-9 [45].

\section{Results \\ Study population}

A summary of participant characteristics is shown in Table 1. The total number of participants was 201 dyads (persons living with mild $\mathrm{AD}$ and their informal caregivers). Notably, $48 \%(n=90)$ of informal caregivers did not specify their relationship to the person with $\mathrm{AD}$ (Table 1).

\section{Missing value analysis}

For the DEMQOL, with its 28 items and with 201 participants, there were seven missing responses $(0.1 \%)$, while in the proxy version of the DEMQOL (31 items), there were eleven missing responses $(0.2 \%$; Table 2$)$. The missing values corresponded to seven cases of one missing 
Table 1 Participant characteristics

\begin{tabular}{|c|c|}
\hline Characteristics & $n=201$ \\
\hline \multicolumn{2}{|l|}{ Persons with dementia } \\
\hline Age (years) & $73.74( \pm 7.92)$ \\
\hline Female & $88(43.8 \%)$ \\
\hline Education level (years) & $12.53( \pm 3.05)$ \\
\hline General cognitive ability (MMSE) & $25.06( \pm 2.17)$ \\
\hline Functional ability (B-ADL, proxy rating) & $3.57( \pm 1.88)^{a}$ \\
\hline Depression (GDS) & $8.90( \pm 5.16)$ \\
\hline Challenging behavior (12 types NPI) & $9.72( \pm 9.30)$ \\
\hline \multicolumn{2}{|l|}{ Informal caregivers (proxy raters) } \\
\hline Age (years) & $64.82( \pm 13.17)$ \\
\hline Female & $143(71.1 \%)$ \\
\hline Spouse of the person with dementia & $80(72.1 \%)^{b}$ \\
\hline Depression (BDI) & $8.35( \pm 6.53)^{a}$ \\
\hline Burden (ZBI-22) & $19.12( \pm 12.52)$ \\
\hline \multicolumn{2}{|c|}{$\begin{array}{l}\text { Data are reported as the mean ( } \pm \text { standard deviation) or number (\%).MMSE } \\
\text { Mini-Mental Status Examination, B-ADL Bayer Activities of Daily Living } \\
\text { Scale;GDS Geriatric Depression Scale, NPI Neuropsychiatric Inventory;BDI Beck } \\
\text { Depression Inventory, ZBI-22 Zarit Burden Interview with } 22 \text { items } \\
\text { a Missing value in } 1 \text { case } \\
\text { b Missing values in } 90 \text { cases }\end{array}$} \\
\hline
\end{tabular}

item (3.6\%) in the DEMQOL, as well as nine cases in which one item (3.2\%) and one case in which two items (6.5\%) were missing in the DEMQOL-Proxy. Ten complete datasets with two-way imputed integers for missing data were generated for the MSA.

\section{Item distribution}

Five items $(20-22,25,26)$ for the DEMQOL and six items (21-24, 28, 29) for the DEMQOL-Proxy showed a ceiling effect (Table 2). The 15 identical items of the DEMQOL and $D E M Q O L$-Proxy demonstrated that proxy ratings are typically lower than the corresponding self-ratings (Table 2).

\section{Structural validity}

Four cores were found for the DEMQOL: items $21 / 24$, $7 / 9,17 / 19$, and $6 / 10\left(\right.$ all $\mathrm{H}_{\mathrm{ij}} \geq 0.49$ ). In addition, for the DEMQOL-Proxy, four cores were found, consisting of items $4 / 8,12 / 14,5 / 7$, and $23 / 24\left(\right.$ all $\left.\mathrm{H}_{\mathrm{ij}} \geq 0.65\right)$. The results of the iterative MSA are shown in Table 3 . We found four equal subscales for both instruments, i.e., [A] "positive emotions", [B] "negative emotions", [C] "physical and cognitive functioning", and [D] "daily activities and social relationships" (Table 3).

For the DEMQOL, the $\mathrm{H}^{\mathrm{S}}$ coefficient showed a "medium" correlation for three subscales $(\mathrm{H}=0.42-0.46)$ in comparison to the subscale $[\mathrm{A}]$, which presented only a "weak" $(\mathrm{H}$ $=0.37$ ) correlation. The correlations of the DEMQOL-Proxy subscales are "strong" ([A] $\left.\mathrm{H}^{\mathrm{S}}=0.66\right)$ and "medium" subscales $\left([C] \mathrm{H}^{\mathrm{S}}=0.50 ;[\mathrm{B}] \mathrm{H}^{\mathrm{S}}=0.46\right.$; [D] $\left.\mathrm{H}^{\mathrm{S}}=0.42\right)$. Subscale [A] differed between both instruments, with only a "weak"
$\mathrm{H}^{\mathrm{S}}$ in the DEMQOL but a "strong" $\mathrm{H}^{\mathrm{S}}$ in the DEMQOLProxy version. The MSA used all items of the proxy version, while in the DEMQOL, two items (11: "irritable"; 15: "forgetting who people are") could not be assigned. With regard to content, item 11 could have matched to subscale [B], while item 15 would have matched to subscale [C]. However, both showed a $\mathrm{H}_{\mathrm{i}}$ of $<0.3$. Item 24 from the $D E M Q O L$, "making yourself understood", and the corresponding item 20 of the DEMQOL-Proxy version, "making him/herself understood", are in different subscales ([C] vs. [D]). Apart from this finding and the missing item 11 in the $D E M Q O L$ version, the remaining 13 of the 15 identical items of the two instruments have been assigned to the same subscales. In the DEMQOL, the item 18 ("difficulty making decisions") presented as a cross loader, which could have assigned to subscale [C] with $\mathrm{H}_{\mathrm{i}}=0.45$ as well as to subscale [D] with $\mathrm{H}_{\mathrm{i}}=0.39$. For a better fit with respect to content and the conceptual framework of Smith et al. [15], we decided to move item 18 into subscale [C]. The assumption of monotonicity for the MSA has been achieved for each of the ten imputed samples for all items $($ Crit $=0)$. All items with a ceiling effect could be found in subscale [D].

Sensitivity analyses were carried out to determine the stability of the identified subscales using the data from the CORDIAL study follow-up surveys, which can be found in Additional file 1: Table $\mathrm{A} 1$ for time $\mathrm{T}_{1}$ after three months and in Additional file 2: Table A2 for the nine-month survey $\left(\mathrm{T}_{2}\right)$ in the supplemental material of this article. With the exception of item 26 in the DEM$Q O L$ version at time $\mathrm{T}_{2}$, the results were confirmed. The $\mathrm{H}^{\mathrm{S}}$ values in the $D E M Q O L$ version are comparable to the baseline values with "medium" correlations for subscale $[B]$ to $[D]$ and to a "weak" correlation for subscale [A] (Additional file 1: Table A1). At $\mathrm{T}_{2}$, the assessment on subscale [A] improved to a "medium", whereas subscale [D] marginally worsened. The subscales [B] and [C] of the DEMQOL-Proxy improved to "strong" for both timepoints. The same result was found for subscale [D] at $\mathrm{T}_{2}$. Therefore, at $\mathrm{T}_{2}$, all subscales of the DEMQOL-Proxy are "strong" (Additional file 2: Table A2).

\section{Internal consistency}

The subscales revealed a $\rho$ of $0.71-0.84(\alpha=0.72-0.83)$ for the DEMQOL and a $\rho$ of $0.79-0.88(\alpha=0.81-0.87)$ for the $D E M Q O L$-Proxy. According to the $\rho$ - and $\alpha$-values, all subscales were considered "good" (Table 3). The internal consistency was also "good" for $\mathrm{T}_{1}$ and $\mathrm{T}_{2}$ for all subscales, with $\rho$ - and $\alpha$-values $>0.72$ (Additional file 1: Table A1 and Additional file 2: Table A2). The $r_{i t}$ revealed only one item (item 9 from the DEMQOL-Proxy, "irritable") that was below 0.4 , which was not scalable at all in the DEMQOL version. All other items had a "high" $(n=41)$ or "moderate" $(n=15) r_{i t}$-values of 0.4 or more. 


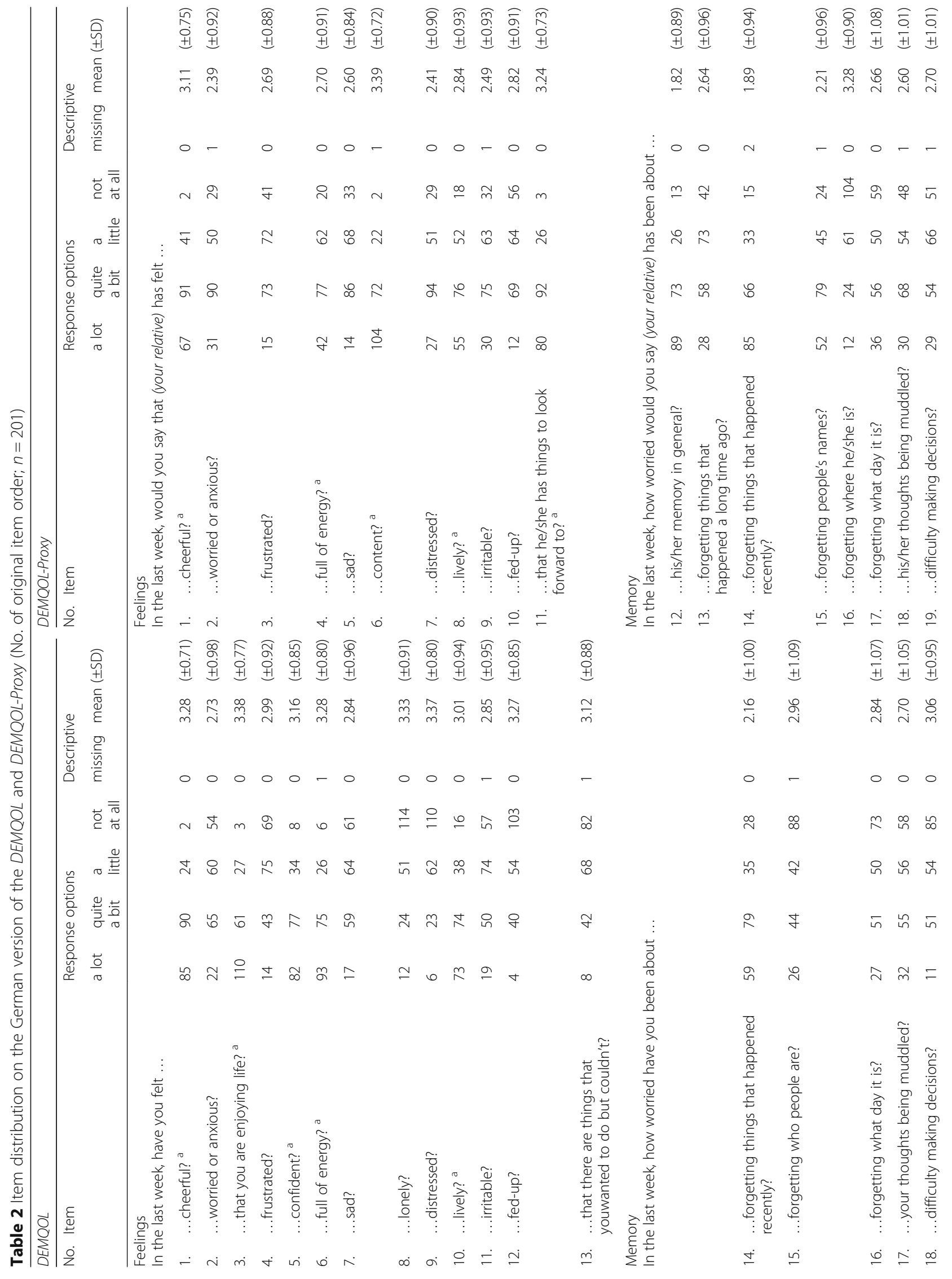




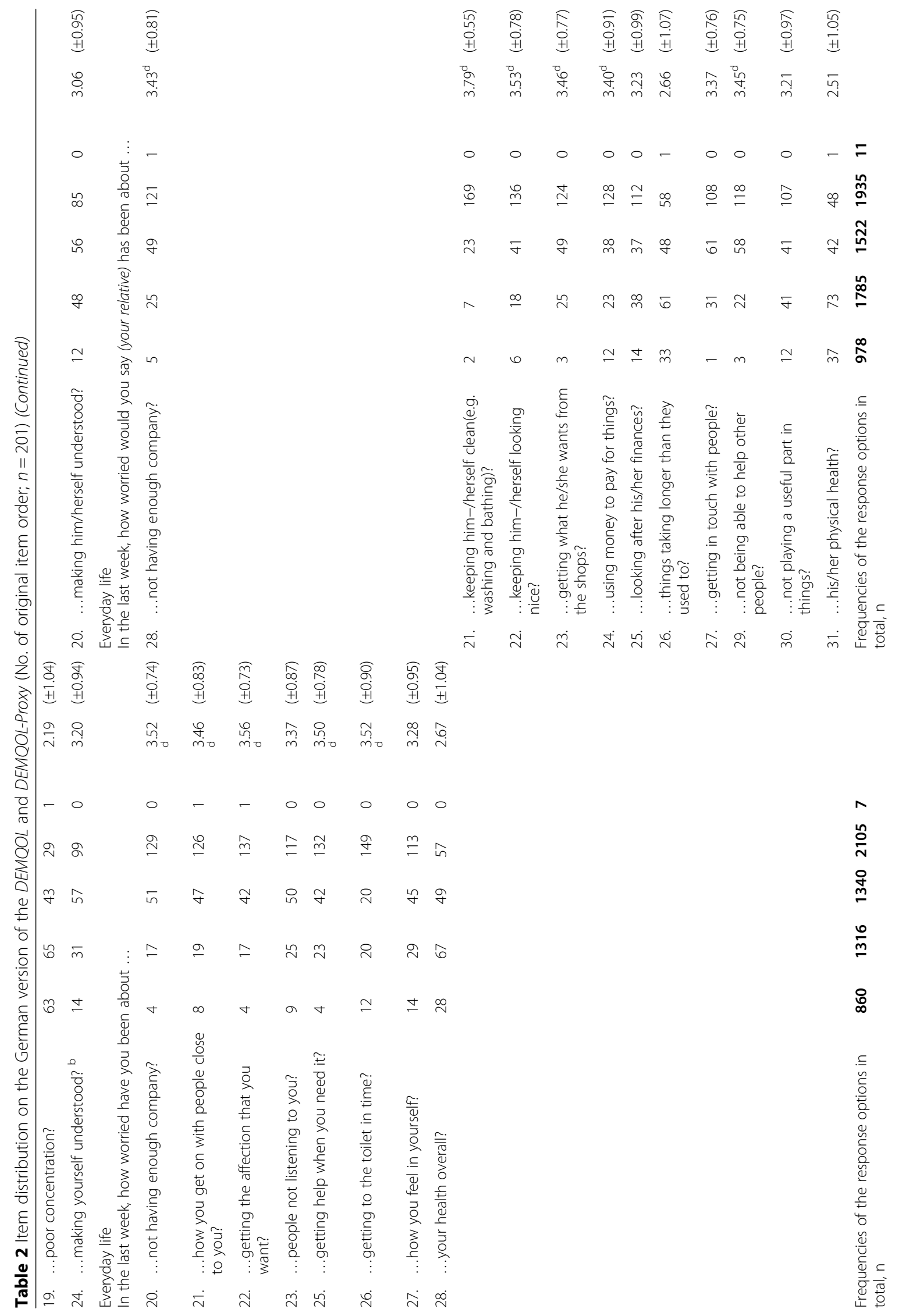




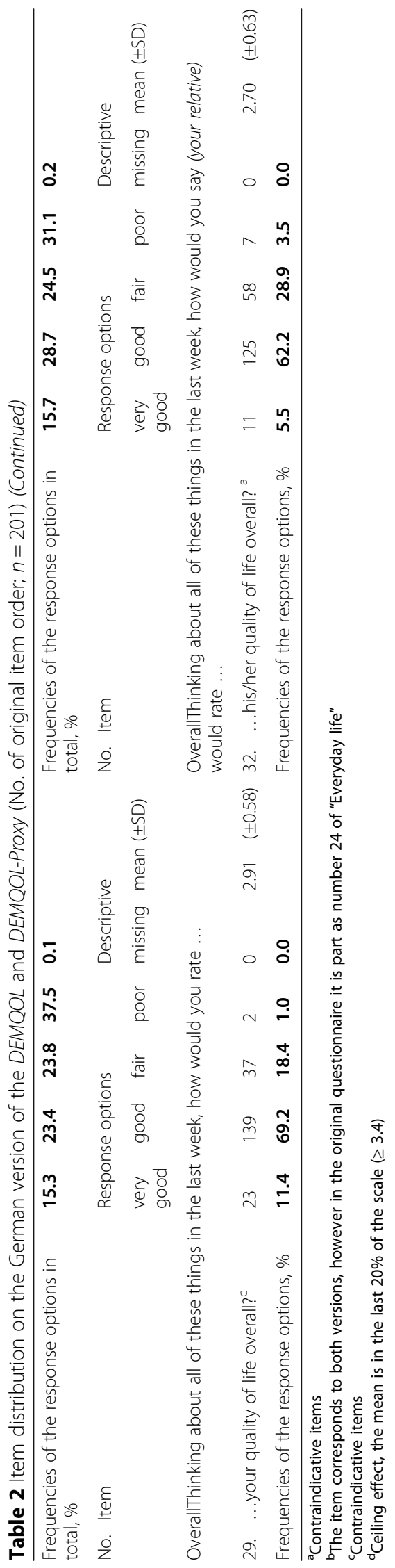


Table 3 Scale analysis and internal consistency of the DEMQOL and DEMQOL-Proxy $(n=201)$

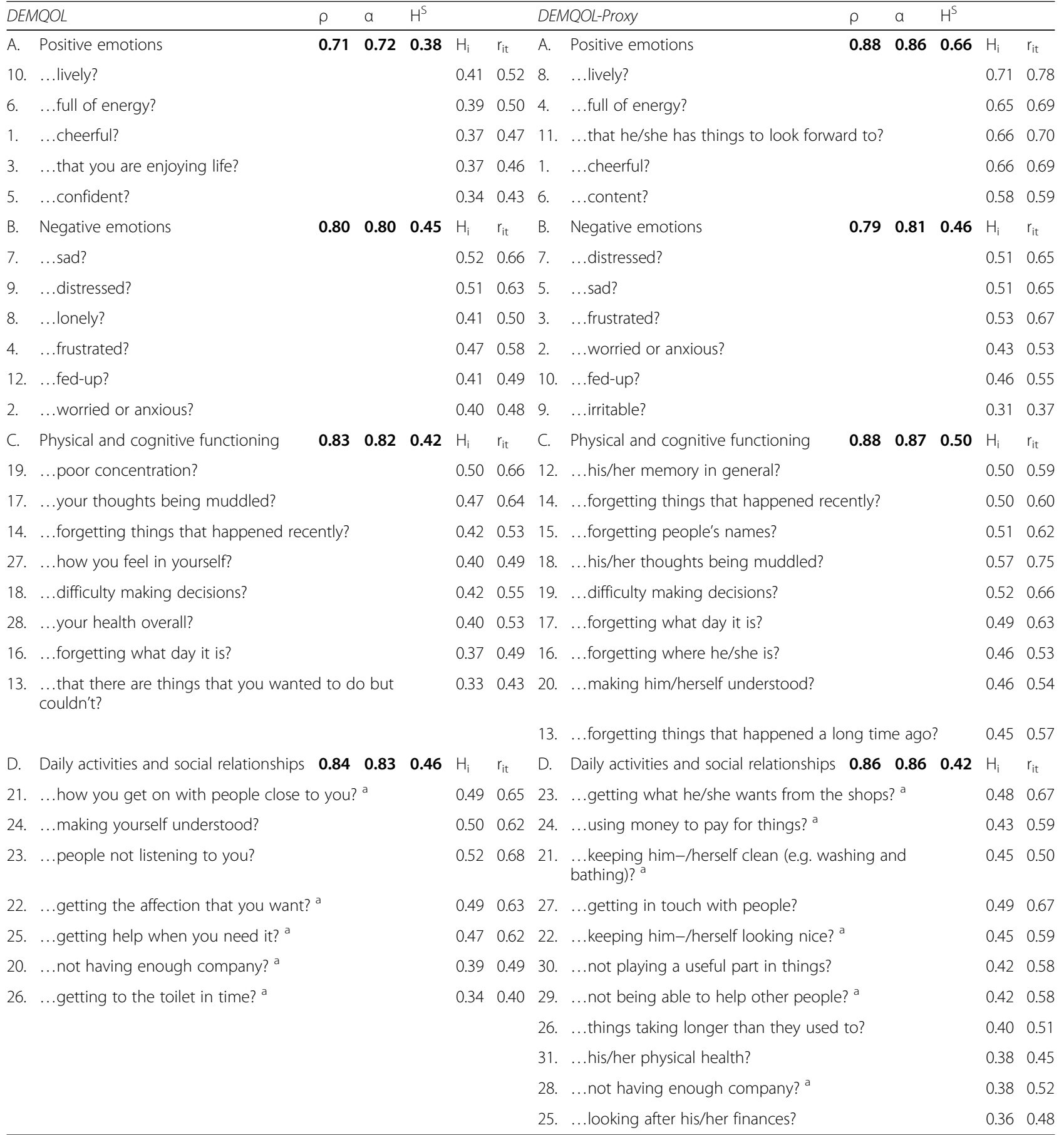

The criterion (Crit) by Molenaar, Sijtsma, and Boer used to check the monotonicity assumption is for all items in every of the ten imputed datasets $=0$ $\rho=$ rho (Molenaar Sijtsma statistic); $a=$ Cronbach's alpha ( $>0.7$ and $<0.95=$ good); $r_{\text {it }}=$ part-whole-corrected item-total correlation ( $>0.50=$ high,$>0.30=$ moderate);

$\mathrm{H}^{\mathrm{S}}=$ Loevinger's $\mathrm{H}$ coefficient of scalability of the scale $\left(>0.50=\right.$ strong, $>0.40=$ medium, $>0.30=$ weak); $H_{\mathrm{i}}=$ Loevinger's $\mathrm{H}$ coefficient for an item and the remaining items of the scale

a Item with ceiling effect (see Table 2)

\section{Discussion}

The evaluation of the German versions of the DEM$Q O L$ and DEMQOL-Proxy revealed the following major findings. First, the MSA for the DEMQOL and the DEMQOL-Proxy revealed that four subscales ([A] "positive emotions", [B] "negative emotions", [C] "physical and cognitive functioning", and [D] "daily activities and social relationships") were found with 
stable $H^{S}$ values $\geq 0.38$. Second, the internal consistency showed consistently "good" values. Third, the item description showed ceiling effects, as exhibited by $18 \%$ of the items on the DEMQOL and $19 \%$ of the items on the DEMQOL-Proxy. Furthermore, the results of the MSA for the German versions of the DEMQOL and DEMQOL-Proxy are largely comparable to findings from previous studies on the HRQoL of persons living with dementia [4, 15], especially to the conceptual framework of Smith and colleagues [14] as well as to their explorative factor analysis of the pretest during the development of the instrument. As such, our results can be considered to be first indications of the structural validity of the $D E M Q O L$ and the DEMQOL-Proxy.

In our data evaluation, the domain "health and well-being" of the conceptual framework is represented by subscales [A], "positive emotions", and [B], "negative emotions", except items 27 ("how you feel in yourself") and 28 ("your health overall"), which have been loaded onto subscale $[\mathrm{C}]$, "physical and cognitive functioning". Therefore, this subscale has been complemented by the word "physical". For the DEMQOL-Proxy, only item 31 ("his/her physical health") from the framework was loaded onto subscale [D], "daily activities and social relationships". Subscale $[C]$ characterizes the domain "cognitive functioning" of the conceptual framework in our data evaluation. Herein, $100 \%$ conformity for the DEMQOL-Proxy could be shown. However, item 24 of the $D E M Q O L$ ("making yourself understood") loaded onto subscale [D]. The domains "daily activities" and "social relationships" of the framework were combined into the fourth subscale of our study, which was designated as subscale [D], "daily activities and social relationships". However, item 13 of the DEMQOL ("that there are things that you wanted to do but couldn't") was an exception, as it was loaded to the subscale $[\mathrm{C}]$, "physical and cognitive functioning". This loading may be due to the translation of the word "inability" into German. Furthermore, item 30 of the DEMQOLProxy ("not playing a useful part in things") was added to subscale [D]. This was the only item that could be assigned to the "self-concept" domain of the conceptual framework.

Compared to the findings of Mulhern and colleagues, we also found subscales for "positive emotions" and "negative emotions" in our study. In contrast, in our assessment, subscale $[C]$, "physical and cognitive functioning", is related to what has been termed "cognition" in the HTA report by Mulhern and colleagues [4]. The subscales "social relationships" in the $D E M Q O L$ and "daily activities" in the DEMQOL-Proxy were referred to the common subscale [D], "daily activities and social relationships", for both instruments within our data evaluation. Taken together, $71 \%$ of the DEMQOL items and $65 \%$ of the DEMQOL-Proxy items coincided with the results of Mulhern et al. [4]; that is, 20 items that are equally distributed in both instruments.

Furthermore, the subscales "positive emotions" (both instruments) and "negative emotions" (DEMQOL-Proxy only) are completely identical, if the cross loaders of the DEMQOL-Proxy in the Mulhern study are not removed. Subscale [B] of the DEMQOL, "negative emotions", exhibits a difference, as given by the loading of items 8 and 9, while item 11 is not loaded. In contrast, the subscale "cognition" shows high consistencies with the $D E M Q O L$ and DEMQOL-Proxy. Differences in the $D E M Q O L$, however, exist only in the additional loading of the items 13, 27 and 28, while item 15 was not loaded. On the DEMQOL-Proxy, item 26 was not loaded, while all other items were identical. Item 20 of $D E M Q O L$ was additionally loaded within subscale [D], "daily activities and social relationships", which was named "social relationships" by Mulhern et al. [4]. Only three items of the DEMQOL-Proxy could be seen in the data set of Mulhern et al. [4], while our data evaluation further loaded the items 21, 22, and 26-31.

In summary, our data demonstrated the determined subscales to be highly consistent with the conceptual framework of Smith et al. [15] and that these subscales further exhibit similarities to those of Mulhern et al. [4]. The sensitivity analysis at times $T_{1}$ and $T_{2}$ showed a stable result for assignment of the items to the subscales (Additional file 1: Table A1 and Additional file 2: Table A2). In contrast, the designation of subscale [D] was identical in the DEMQOL and DEMQOL-Proxy, while this finding differed according to Mulhern et al. [4] and was therefore presented as "social relationships" in the $D E M Q O L$ and "daily activities" in the DEMQOL-Proxy. However, if both subscales were taken together, they represent a similar construct as our subscale [D], "daily activities and social relationships".

In accordance with the HRQoL definition from Hays and Reeve [7] provided in the background, the subscales we found in our study cover the aspects of "how well a person functions in his/her life" (physical and cognitive functioning $[\mathrm{C}]$ ) and his or her "perceived well-being in physical (daily activities), mental (positive and negative emotions $[\mathrm{A}-\mathrm{B}]$ ), and social domains (social relationships [D]) of health".

\section{Limitations}

The presented data of this psychometric study used datasets from the CORDIAL study by Kurz et al. [21]. Thus, since additional data could not be obtained, no statements on the inter-rater reliability of the $D E M$ QOL-Proxy can be made to estimate the quality of the 
underlying data. Similarly, it was no longer possible to influence the number of study participants. According to a study by Straat et al. [46], a sample of more than 250 respondents should be given if the quality of the answers is high. In the present study, however, only the data of 201 persons could be used, reflecting a limitation of the results. Furthermore, the CORDIAL study included only persons with a mild severity of AD. The mild form might explain the ceiling effects of subscale [D]. Thus, the generalizability of the results is limited due to the absence of other forms of dementia.

\section{Conclusions}

In this psychometric study using the German versions of the instruments DEMQOL and DEMQOL-Proxy, four equal subscales were found in both instruments, demonstrating "good" internal consistency. The subscales reflect the conceptual framework of the instrument developers to a high degree. Thus, the results can be considered a first indication of the construct validity of the two German versions. In our opinion, DEMQOL subscale scores are more explanatory than a total score because HRQoL is a multidimensional concept and respective domain scores may help clarify treatment impacts. Moreover, our internal consistency results reflect the homogeneity of the subscales. However, Chua et al. [19] used bifactor models for direct comparisons between total and subscale scores and showed that the latter scores had poor reliability and should not be used. Such direct comparisons were not performed in our study due to differences in modeling decisions (parallel iterative MSA rather than bifactor modeling). The merits of MSA and bifactor modeling for clarifying multidimensionality are debatable [47, 48]. Therefore, more empirical data are needed before definitive recommendations can be made.

However, we recommend further investigations prior to or integrated in future studies using the German version of the DEMQOL and DEMQOL-Proxy. In particular, we advise the integration of the evaluation of test-retest reliability (both $D E M Q O L$ versions) and inter-rater reliability (DEMQOL-Proxy) as part of the instrument application in future studies. Continued research should also be carried out on structural validity using various latent variable models. In addition, further investigation of data from persons living with moderate dementia, or with severe dementia for the proxy version, should be performed according to the ceiling effects we found in our study. For the proxy version, it would also be important to conduct a study with professional nurses to make statements on the use of the instrument in German nursing homes. Similarly, an investigation should be conducted to analyze against external criteria for other proportions of the construct validity.

\section{Additional files}

Additional file 1: Table A1. Scale analysis and internal consistency of the DEMQOL $(n=183)$ and DEMQOL-Proxy $(n=179)$ using the data of $\mathrm{T}_{1}$, three months after the baseline assessment. (PDF $240 \mathrm{~kb}$ )

Additional file 2: Table A2. Scale analysis and internal consistency of the DEMQOL $(n=166)$ and DEMQOL-Proxy $(n=163)$ using the data of $T_{2}$, nine months after the baseline assessment. (PDF $316 \mathrm{~kb}$ )

\section{Abbreviations}

AD: Alzheimer's disease; B-ADL: Bayer Activities of Daily Living Scale; BDI: Beck Depression Inventory; CORDIAL: Cognitive Rehabilitation and Cognitive Behavioral Treatment for Early Dementia in Alzheimer's Disease (study); Crit: Criterion by Molenaar, Sijtsma, and Boer; DEMQOL: Dementia Quality of Life; GDS: Geriatric Depression Scale; $\mathrm{H}_{i}$ : Loevinger's H coefficient for an item and the remaining items of the scale; HRQoL: Health-related quality of life; $H^{S}$ : Loevinger's $H$ coefficient of scalability of a scale;

HTA: Health Technology Assessment; ICF: International Classification of Functioning, Disability and Health; MMSE: Mini-Mental Status Examination; MSA: Mokken scale analysis; NPI: Neuropsychiatric Inventory; QoL: Quality of life; rit: Part-whole-corrected item-total correlation; SD: Standard deviation; WHO: World Health Organization; ZBI-22: Zarit Burden Interview with 22 items; a: Cronbach's alpha; p: rho (Molenaar Sijtsma statistic)

\section{Acknowledgments}

We would like to thank the participants and colleagues from the CORDIAL study. We would especially like to thank Professor Dr. Alexander Kurz for providing the data.

\section{Ethical approval and consent to participate}

The study protocol was approved by the ethics committees of the coordination center and the participating recruitment sites [21]. Ethical clearance was granted for the CORDIAL study by the Ethics Commission of the Faculty of Medicine of the Technical University of Munich on 12/10/2009 (no. 2113/08 S). The participants received written and oral information about the study prior to data collection. Written informed consent was obtained from both the persons living with $A D$ and their informal caregivers. We refrained from re-auditing as ethical clearance is not required for analyses based on secondary data [22] or for studies using anonymous data.

\section{Funding}

The German Federal Ministry of Health funded the CORDIAL study (2008-2010; funding code: IIA5-2508FSB105//44-034). This study was also a "lighthouse project dementia". The German Center for Neurodegenerative Diseases supported the data analysis for this article. Both funding institutions had no role in the design, conduct, analyses or reporting of this study.

\section{Availability of data and materials}

All of the data necessary for a meta-analysis of the internal consistency and structural validity of the DEMQOL and DEMQOL-Proxy items are contained within the manuscript and its supplementary file. Further data are available upon request.

\section{Authors' contributions}

Study concept: CGGS, MND, and MB. Data collection and handling: CGGS and MB. Data analysis: CGGS and MND. Manuscript preparation: CGGS, MND, and MB. All authors have read and approved the final version of the manuscript.

\section{Consent for publication}

Not applicable.

\section{Competing interests}

The authors declare that they have no competing interests.

\section{Publisher's Note}

Springer Nature remains neutral with regard to jurisdictional claims in published maps and institutional affiliations. 


\section{Author details}

${ }^{1}$ German Center for Neurodegenerative Diseases (DZNE), Stockumer Straße 12, 58453 Witten, Germany. ${ }^{2}$ School of Nursing Science, Witten/Herdecke University, Stockumer Straße 12, 58453 Witten, Germany. ${ }^{3}$ Clinic for Cognitive Neurology, Medical Faculty, Leipzig University, Liebigstraße 16, 04103 Leipzig, Germany.

\section{Received: 5 September 2017 Accepted: 26 September 2018}

\section{Published online: 19 October 2018}

\section{References}

1. Alzheimer's Disease International (ADI). World Alzheimer Report 2015: The global impact of dementia. In London; 2015.

2. American Psychiatric Association (ed.): DSM-5. Diagnostic and statistical manual of mental disorders, Fifth Edition edn. Washington/DC: American Psychiatric Publishing; 2013.

3. Guidelines on medicinal products for the treatment of Alzheimer's disease and other dementias [http://www.ema.europa.eu/docs/en_GB/document_ library/Scientific_guideline/2009/09/WC500003562.pdf].

4. Mulhern B, Rowen D, Brazier J, Smith S, Romeo R, Tait R, Watchurst C, Chua KC, Loftus V, Young T et al: Development of DEMQOL-U and DEMQOLPROXY-U: generation of preference-based indices from DEMQOL and DEMQOL-PROXY for use in economic evaluation. Health Technology Assessment 2013, 17(5):v-xv, 1-140.

5. Rowen D, Mulhern B, Banerjee S, Hout B, Young TA, Knapp M, Smith SC, Lamping DL, Brazier JE. Estimating preference-based single index measures for dementia using DEMQOL and DEMQOL-Proxy. Value Health. 2012;15. United States:346-56.

6. Rowen D, Mulhern B, Banerjee S, Tait R, Watchurst C, Smith SC, Young TA, Knapp M, Brazier JE. Comparison of general population, patient, and carer utility values for dementia health states. Medical Decision Making. 2015;35. United States:68-80.

7. Hays RD, Reeve BB: Measurement and modeling of health-related quality of life. In: Epidemiology and demography in public health. Edited by Killewo J, Heggenhougen HK, Quah SR. San Diego CA: Academic Press; 2010: 195-205.

8. Karimi M, Brazier J. Health, health-related quality of life, and quality of life: what is the difference? PharmacoEconomics. 2016;34(7):645-9.

9. Wilson IB, Cleary PD. Linking clinical variables with health-related quality of life. A conceptual model of patient outcomes. Jama. 1995;273(1):59-65.

10. World Health Organization (WHO). Constitution of the World Health Organization. Basic documents of the Word Helath Organization. Geneva: World Health Organization; 2014.

11. Bakas T, McLennon SM, Carpenter JS, Buelow JM, Otte JL, Hanna KM, Ellett ML, Hadler KA, Welch JL. Systematic review of health-related quality of life models. Health Qual Life Outcomes. 2012;10:134.

12. World Health Organization (WHO). International Classification of Functioning, disability, and Health: Children and Youth Version: ICF-CY. Geneva: World Health Organization; 2007.

13. Ferrans CE, Zerwic JJ, Wilbur JE, Larson JL. Conceptual model of health-related quality of life. Journal of nursing scholarship : an official publication of Sigma Theta Tau International Honor Society of Nursing. 2005;37(4):336-42.

14. Smith SC, Murray J, Banerjee S, Foley B, Cook JC, Lamping DL, Prince M, Harwood RH, Levin E, Mann A. What constitutes health-related quality of life in dementia? Development of a conceptual framework for people with dementia and their carers. International journal of geriatric psychiatry. 2005;20(9):889-95.

15. Smith SC, Lamping DL, Banerjee S, Harwood R, Foley B, Smith P, Cook JC, Murray J, Prince $\mathrm{M}$, Levin $\mathrm{E}$, et al. Measurement of health-related quality of life for people with dementia: development of a new instrument (DEMQOL) and an evaluation of current methodology. Health Technol Assess. 2005;9(10):1-93 iii-iv.

16. Berwig M, Leicht $\mathrm{H}$, Gertz HJ. Critical evaluation of self-rated quality of life in mild cognitive impairment and Alzheimer's disease - further evidence for the impact of anosognosia and global cognitive impairment. The journal of nutrition health and aging. 2009;13(3):226-30.

17. Dichter MN, Schwab CGG, Meyer G, Bartholomeyczik S, Halek M. Linguistic validation and reliability properties are weak investigated of most dementiaspecific quality of life measurements - a systematic review. J Clin Epidemiol. 2016;70:233-45

18. Lucas-Carrasco R, Lamping DL, Banerjee S, Rejas J, Smith SC, Gomez-Benito $J$. Validation of the Spanish version of the DEMQOL system. International psychogeriatrics / IPA. 2010;22(4):589-97.
19. Chua KC, Brown A, Little R, Matthews D, Morton L, Loftus V, Watchurst C, Tait R, Romeo R, Banerjee S. Quality-of-life assessment in dementia: the use of DEMQOL and DEMQOL-proxy total scores. Quality of life research : an international journal of quality of life aspects of treatment, care and rehabilitation. 2016;25(12):3107-18.

20. Hendriks AAJ, Smith SC, Chrysanthaki T, Cano SJ, Black N. DEMQOL and DEMQOL-proxy: a Rasch analysis. Health Qual Life Outcomes. 2017;15(1):164.

21. Kurz A, Thone-Otto A, Cramer B, Egert S, Frolich L, Gertz HJ, Kehl V, Wagenpfeil S, Werheid K. CORDIAL: cognitive rehabilitation and cognitivebehavioral treatment for early dementia in Alzheimer disease: a multicenter, randomized, controlled trial. Alzheimer Dis Assoc Disord. 2012;26(3):246-53.

22. Swart E, Gothe H, Geyer S, Jaunzeme J, Maier B, Grobe TG, Ihle P, et al. [Good Practice of Secondary Data Analysis (GPS): Guidelines and Recommendations] Gute Praxis Sekundärdatenanalyse (GPS): Leitlinien und Empfehlungen. Gesundheitswesen. 2015;77(02):120-6.

23. Kessler J, Markowitsch HJ, Denzler PE. [Mini-Mental State Examination (MMSE) - German version] Mini-Mental-Status-Test (MMSE) - Deutsche Fassung. Beltz: Weinheim; 1990.

24. Hindmarch I, Lehfeld $H$, de Jongh P, Erzigkeit $H$. The Bayer activities of daily living scale (B-ADL). Dement Geriatr Cogn Disord. 1998;9(suppl 2):20-6.

25. Yesavage JA. Geriatric depression scale. Psychopharmacol Bull. 1988;24(4): 709-11.

26. Cummings $\mathrm{JL}$. The neuropsychiatric inventory: assessing psychopathology in dementia patients. Neurology. 1997;48(5 Suppl 6):10-6.

27. Hautzinger M, Bailer M, Worall H, Keller F: [Beck-depression-inventory (BDI): editing of the German version - test manual] Beck-depressions-Inventar (BDI): Bearbeitung der deutschen Ausgabe - Testhandbuch, 2. Auflage edn. Bern: Hans Huber; 1994.

28. Zarit SH, Reever KE, Bach-Peterson J. Relatives of the impaired elderly: correlates of feelings of burden. The Gerontologist. 1980;20(6):649-55.

29. Stochl J, Jones PB, Croudace TJ. Mokken scale analysis of mental health and wellbeing questionnaire item responses: a non-parametric IRT method in empirical research for applied health researchers. BMC Med Res Methodol. 2012;12:74.

30. Watson R, van der Ark LA, Lin LC, Fieo R, Deary IJ, Meijer RR. Item response theory: how Mokken scaling can be used in clinical practice. I Clin Nurs. 2012;21(19-20):2736-46.

31. Dichter MN, Dortmann O, Halek M, Meyer G, Holle D, Nordheim J, Bartholomeyczik S. Scalability and internal consistency of the German version of the dementia-specific quality of life instrument QUALIDEM in nursing homes -- a secondary data analysis. Health Qual Life Outcomes. 2013;11(1):91.

32. Bouman Al, Ettema TP, Wetzels RB, van Beek AP, de Lange J, Droes RM. Evaluation of Qualidem: a dementia-specific quality of life instrument for persons with dementia in residential settings; scalability and reliability of subscales in four Dutch field surveys. International journal of geriatric psychiatry. 2011;26(7):711-22.

33. Sijtsma K, Molenaar IW. Introduction to nonparametric item response theory, vol. 5. Thousand Oaks, CA: Sage Publications; 2002.

34. Molenaar IW, Sijtsma K. User's manual MSP5 for windows [software manual]. IEC ProGAMMA: Groningen, The Netherlands; 2000.

35. Müller-Schneider T. Multiple Skalierung nach dem Kristallisationsprinzip. Eine alternative zur explorativen Faktorenanalyse [multiple scaling according to the principle of crystallization. An alternative method to exploratory factor analysis]. Z Soziol. 2001;30(4):305-15.

36. Schwab CGG. [Construct validity and internal consistency of the instrument "burden in dealing with dementia" (BelaDem) in nursing homes unpublished master thesis] Konstruktvalidität und interne Konsistenz des Instrumentes "Belastungserleben im Umgang mit Demenz" (BelaDem) in der stationären Altenhilfe - unveröffentlichte Masterarbeit. Herdecke: Witten: Universität Witten/Herdecke; 2013.

37. Van Ginkel JR, Andries van Der Ark LA, Sijtsma K, Vermunt JK. two-way imputation: a Bayesian method for estimating missing scores in tests and questionnaires, and an accurate approximation. Computational Statistics \& Data Analysis. 2007;51(8):4013-27.

38. Sijtsma K, Molenaar IW. Reliability of test scores in nonparametric item response theory. Psychometrika. 1987;52.

39. van der Ark LA, van der Palm DW, Sijtsma K. A latent class approach to estimating test-score reliability. Appl Psychol Meas. 2011;35(5):380-92.

40. Terwee CB, Bot SD, de Boer MR, van der Windt DA, Knol DL, Dekker J, Bouter LM, de Vet HC. Quality criteria were proposed for measurement properties of health status questionnaires. J Clin Epidemiol. 2007;60(1):34-42. 
41. Bortz J, Döring N, Bortz D. [Research methods and evaluation: for human and social sciences] Forschungsmethoden und Evaluation: für Human- und Sozialwissenschaftler, fourth revised edition edn. Heidelberg: SpringerMedizin-Verlag; 2006

42. Core R. Team R: a language and environment for statistical computing. Vienna, Austria: R Foundation for Statistical Computing; 2017.

43. van der Ark LA. Mokken scale analysis in R. J Stat Softw. 2007;20(11):19.

44. van der Ark LA. New developments in Mokken scale analysis in R. J Stat Softw. 2012;48(5):27.

45. Robitzsch A, Grund S, Henke T: TAM: Some additional multiple imputation functions, especially for mice. R package version 2.5-9. https://CRAN.Rproject.org/package=miceadds; 2017.

46. Straat $J H$, van der Ark LA, Klaas $\mathrm{S}$. minimum sample size requirements for Mokken scale analysis. Educ Psychol Meas. 2014;74(5):809-22.

47. Reise SP, Kim DS, Mansolf M, Widaman KF. Is the Bifactor model a better model or is it just better at modeling implausible responses? Application of iteratively reweighted least squares to the Rosenberg self-esteem scale. Multivariate Behav Res. 2016;51(6):818-38.

48. Smits IAM, Timmerman ME, Meijer RR. Exploratory Mokken scale analysis as a dimensionality assessment tool: why scalability does not imply Unidimensionality. Appl Psychol Meas. 2012;36(6):516-39.

Ready to submit your research? Choose BMC and benefit from:

- fast, convenient online submission

- thorough peer review by experienced researchers in your field

- rapid publication on acceptance

- support for research data, including large and complex data types

- gold Open Access which fosters wider collaboration and increased citations

- maximum visibility for your research: over $100 \mathrm{M}$ website views per year

At $\mathrm{BMC}$, research is always in progress.

Learn more biomedcentral.com/submissions 